\title{
Priority Based Prediction Mechanism for Ranking Providers in Federated Cloud Architecture
}

\author{
${ }^{1}$ G.Vadivel, Dr. ${ }^{2}$ M. Aramudhan \\ ${ }^{1}$ Research Scholar, Dept. of CSE, Rayalaseema University Kurnool (A.P), India. \\ ${ }^{2}$ Asst. Prof, Dept. of IT, Perunthalaivar Kamarajar Inst of Engg\&Technology, Nedugadu, Karikal, Puducherry, \\ India.
}

\begin{abstract}
In Current trends the Cloud computing has a lot of potential to help hospitals cut costs, a new report says. Here's some help overcoming some of the challenges hiding in the cloud. Previously there several methods are available for this like Broker based trust architecture and etc. Health care framework which are patient, doctor, symptom and disease. In this paper we are going to discuss the broker based architecture for federated clou d and its Service Measurement Index considered for evaluating the providers,construction of Grade Distribution Table(GDT),concept of ranking the providers based on prediction weights comparison, optimal service provider selection and results discussion compared with existing techniques available for ranking the providers. In this paper we are going to propose, two different ranking mechanis ms to sort the providers and select the optimal provider automatically. Grade distribution ranking model is proposed by assigning the grade for the providers based on the values of SMI attributes, based on the total grade value, providers falls on either Gold, Silver or Bronze. Each category applies the quick sort to sort the providers and find the provider at the top is optimal. If there is more than one provider at the top, apply priority feedback based decision tree and find the optimal provider for the request. In the second ranking mechanis $\mathrm{m}$, joint probability distribution mechanism is used to rank the providers, two providers having same score, apply priority feedback based decision tree and find the optimal provider for the request.
\end{abstract}

Keywords: Cloud computing, priority feedback based decision tree, Grade Distribution Table(GDT), Discovery of service provider.

\section{INTRODUCTION}

Cloud computing is internet-based computing, where shared servers provide computing power, storage, development platforms or software to computers and other devices on demand.

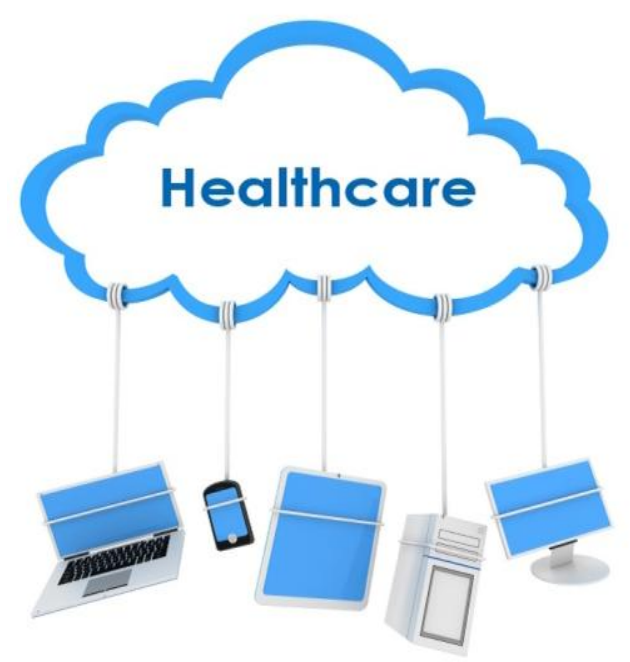

Fig: The Basic Health Cloud Architecture.
This frequently takes the form of cloud services, such as 'Infrastructure as a Service' (IaaS), 'Platform as a Service (PaaS)' or 'Software as a Service' (SaaS). Users can access web-based tools or applications through a web browser or via a cloud-based resource like storage or computer power as if they were installed locally, eliminating the need to install and run the application on the customer's own computers and simplifying maintenance and support. There are several possible deployment models for clouds.

\section{PREVIOUS WORK}

Md Whaiduzzaman, Mohammad Nazmul Haque proposed an method for this cloud health care Analytical hierarchy process (AHP) is a tool for decision makers to be able to do more informed decisions regarding their investment in such technologies. Service measurement index (SMI) is a service measurement model based on business model of the International Standard Organization (ISO).The SMI Cloud model is proposed by Garg et al. [1] which lets users compare different cloud offerings, according to their priorities and along several dimensions, and select whatever is 
appropriate to their needs. Several challenges are tackled in realizing the model for evaluating QoS and ranking cloud providers. The SMICloud systematically measures all the QoS features proposed by cloud service measurement index consortium (CSMIC) and ranks the cloud services based on these business services. Again, Li et al.Thesel problems are solved in our cloud based proposed model. 'Hybrid Clinical Decision Support System for rural Bangladesh' is also a good support system for healthcare. The authors have proposed an automated diagnostic system which could be used in the rural areas where there is a scarcity of doctors .'A Hybrid Mobile-based Patient Location Tracking System for Personal Healthcare Applications' system uses cellular network infrastructure using GPS system for tracking the location of patients. So we have used the idea of GPS in our proposed model to locate patient and find out nearest specialist doctors in surrounding area . NFC based patient appointment system is also useful in our model as our model is flexible and generic so we can add more features also .Rajarajeswari and Aramudhan (2014) proposed a new framework for cloud that maintains the SLA by means of distinguished the incoming requests either SLA based member or SLA based nonmember. Rajkumar Buyya et.al discussed the framework which measures the quality, prioritizes and selects the cloud services based on SMI metrics and ranking the services using Analytic Hierarchy Process (AHP). It is one of the flexible ways for solving and adapted to any number of attributes with any number of sub-attributes. AHP model has three phases such as forming hierarchy structure, pair wise comparisons and find aggregated value to generate ranking of the services. Authors proposed service mapper that contains a technique called singular value decomposition which is used for ranking the services in statistical manner.In all the above methods there is no efficient correlation from symptoms to diseases and diseases to the specialist doctor in previous systems. Our proposed system predict appropriate diseases based on the symptoms and then display the list of specialist doctors in nearby areas.In this proposed federated cloud architecture we are trying to overcome all these drawbacks.

\section{PROPOSED WORK}

The proposed method is developed by based on the Federated cloud architecture it refers to a network of cloud providers that are interconnected based on open standards to provide a universal decentralized computing environment where everything is driven by constraints and agreements. Federated resource provisioning model consists of three phases namely (i) Discovery of service providers (ii) Rank the shortlisted service provider (iii) assigning the service to the best service provider. The customized broker based federated architecture is shown in Figure -1. Broker Manager (BM) collects the various levels of services information offered by cloud service providers through broker learning algorithm. Brokers manage the cloud provider's resources, collect its information and updates in Broker Status Registry (BSR). BM communicates with brokers, discovers the appropriate providers for the requests and short list the providers. Broker based Learning Algorithm (BLA) helps to study the workload of the providers, understand the requirement of resources for the service requests, analysis the suitability of the providers automatically and shortlist it. The clouds are managed by CloudBrokers (CB) that is capable of handling service requests and managing virtual machines within in federate cloud systems. The components of the broker Managers are Differentiated Module (DM), Discovery of the Providers (DP) and Ranking the providers. It must support the management of the collaboration that includes all involved service providers, partners, and end users or consumer. Cloud clients are the users of the cloud services that offer different services towards business goals that driven resource sharing. Application Program Interface (API) acts as an interface point for all kinds of users to interact with services or offerings. This architecture is based on the differential module it was developed in the following steps.

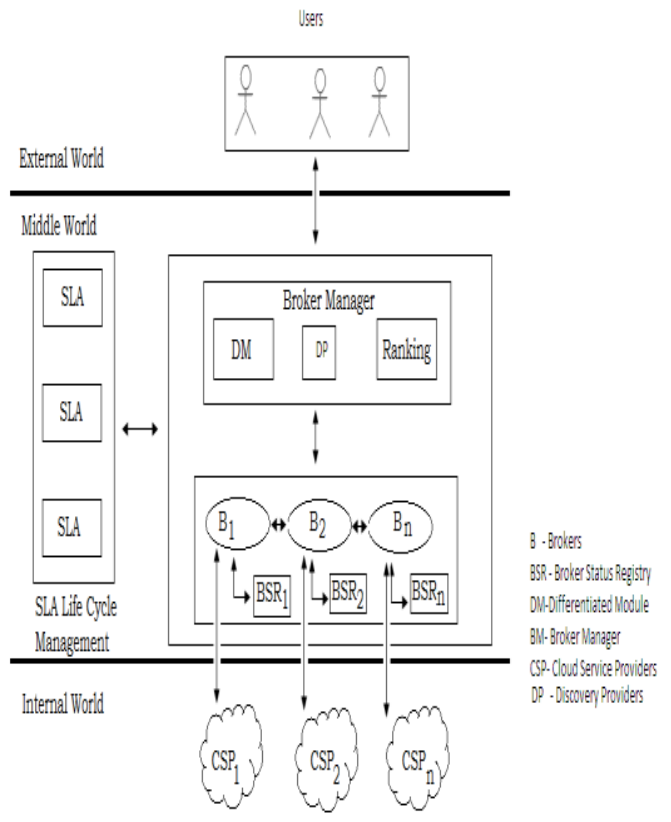

Figure 1: Customized Broker Federated Architecture. 
Step 1: Compute the number of requests in Member and Non- Member queues in the previous session.

Step 2: Calculate the Request Deviation (RD) for each queue using the formula,

$$
\mathrm{RD}_{\mathrm{i}}=\left(\mathrm{n} * \mathrm{~N}_{\mathrm{i}}\right) / \mathrm{N}_{\mathrm{T}} \text {. }
$$

Step 3: Calculate the New Weighting Value (NW V) for each queue if $\left(\mathrm{RD}_{\mathrm{i}}>1\right)$

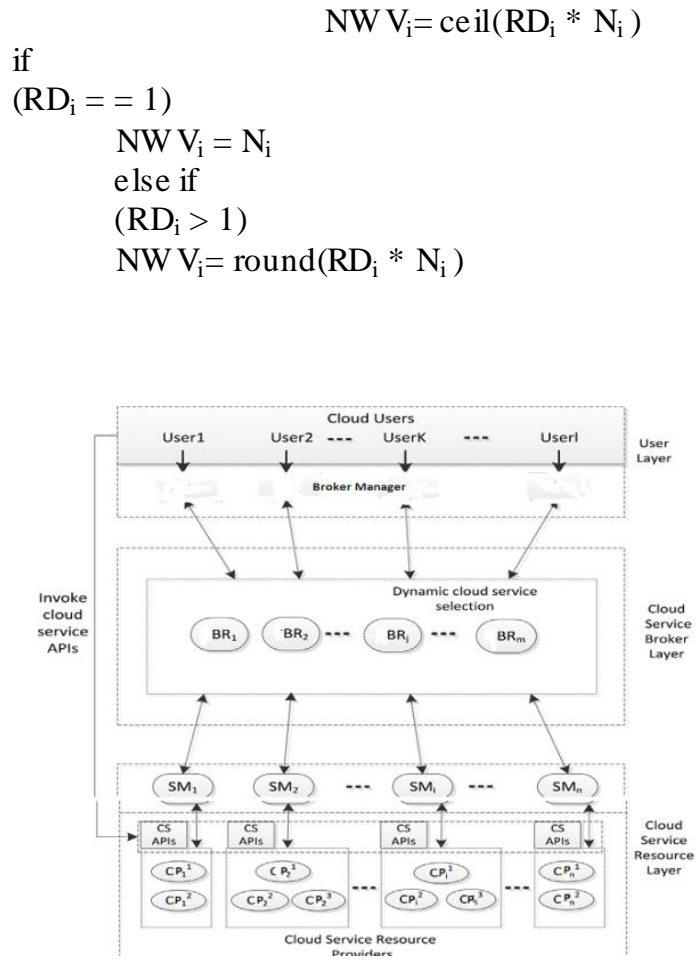

Figure: 2 Discovery of service provider in Federated Architecture.

\section{Service Measurement Index (SMI)}

Service Measurement Index Key Performance Indicators attributes are falling in two types namely measurable and Non-measurable. These attributes are proposed by Cloud Service Measurement Index Consortium (CSMIC) and recognized by International Organization for Standardization (ISO). Non measurable attributes cannot be assigned with numeric value and are mostly inferred based on user experiences. Measurable attributes are those which can be measured using software and hardware monitoring tools. Some of the attributes considered to propose a quality model for provider selection are average response time, reliability, accuracy, service sustainability, stability, adaptability, interoperability, cost.

\section{Grade Distribution Based Ranking Model}

In the proposed model, the grading is assigned for the providers based on the computed values on the considered SMI attributes that used to evaluate the performance of the providers in terms of user, application and service. Three different grading values are considered such as $1,0.5$, and 0.25 . These grade values are distributed to the considered SMI attributes by invoking Grade Distribution Algorithm. After computing the total grade values, the providers may fall on any one of the category namely Gold, Silver and Bronze.

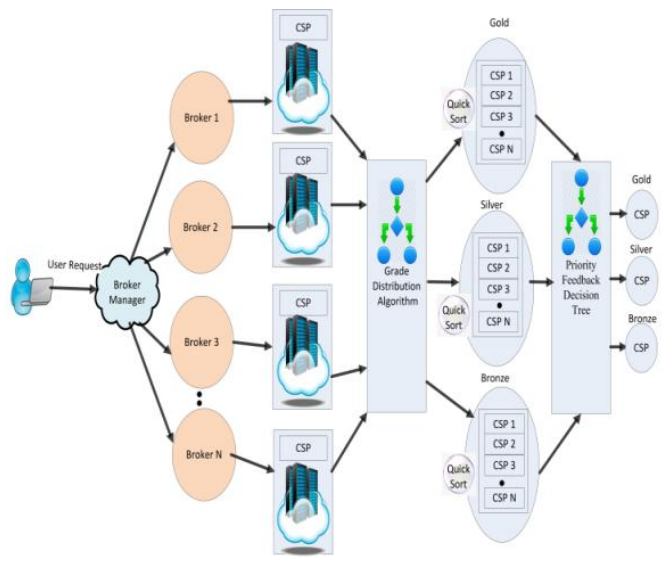

Figure 3: Grade Distribution Based Ranking Model

Grade distribution algorithm works as follows, First case, when availability of the resource is either sufficiently enough or moderately higher to compute the requests, then the value of the weight ' 1 ' is to be assigned to that specific SMI attribute. When the available resource is approximately equal or moderately equal, then the value of the weight ' 0.5 ' is assigned to that specific SMI attribute. Third case, when available resource is very much lower than the request then the value of the weight ' 0.25 ' is assigned to that SMI attribute. In similar way, other considered measurable SMI attributes are distributed with grade values as per the threshold and limit. These tolerate values are not fixed, any time it variable is based on the range of user submission. Based on the total grade values, the providers may be fall on any of the type namely Gold, Silver and Bronze. Quick sort is the best sorting algorithm that placing the providers in order. This algorithm takes $\mathrm{O}$ $(n \log n)$ comparisons to sort $n$ providers. In the worst case, it makes $\mathrm{O}\left(n^{2}\right)$ comparisons, though this behavior is rare, in this work, quick sort technique is used to arrange service providers based on the total grade value. If there is more than one provider in each category having similar total grade value, then selection of the optimal provider is recognized by applying Priority Feedback Decision Tree (PFDT). The working of grade distribution based ranking model is shown in Figure -2. At the last stage of the process, there is a provider in each category. User may select the 
category first and subsequently the provider in the category as the need of the user, application and service requirement. Each category is distinguished with the metric called threshold and limit. Grade based distribution algorithm creates clusters based on threshold value among the short listed pool of providers that attained the fulfillment of the request submitted by the user. Time complexity of the grade distribution algorithm is $\mathrm{O}\left(\mathrm{k}^{*} \mathrm{n} * \mathrm{~d} * \mathrm{i}\right)$ where ' $\mathrm{k}$ 'denotes the number of clusters, ' $\mathrm{n}$ ' refers the number of providers, 'd' denotes the number of considered SMI attributes and 'i' refers the number of iterations needed.

\section{SIMULATION RESULTS \& ANALYSIS}

The proposed ranking mechanism is implemented in CloudSim. The average execution time of members is highly significant to estimate the performance of service providers. To prove that, performance evaluation of the proposed architecture is implemented in CloudSim using Java. The general evaluation parameters considered for the experiment are number of users, number of cloud service providers, load factor of cloud service providers, average load deviation, deadline of tasks etc. The execution time for each task is assigned randomly between $0.1 \mathrm{~ms}$ to $0.5 \mathrm{~ms}$. Number of users considered for the experiment are 1000, 5000 and 10000. Number of service providers available is fixed as 10 , and deadline for each request is fixed as $0.5 \mathrm{~ms}$.Load factor is defined as the ratio between the number of requests in CBM and the number of cloud service providers. Load factor that varies dynamically depends on the number of requests arrived at CBM. The average load deviation is defined as the average difference between the load expected and load assigned to cloud service provider. Performance of cloud service provider decreases when load deviation gets increased. Every cloud service provider consider for the experiment has 50 computing hosts, 10GB of memory, 2TB of storage, 1 processor with 1000 MIPS of capacity, and a time-shared VM scheduler. Cloud broker on behalf of user request consist of $256 \mathrm{MB}$ of memory, $1 \mathrm{~GB}$ of storage, 1 CPU, and time-shared Cloudlet scheduler. The broker requests instantiation of $25 \mathrm{VMs}$ and associates one Cloudlet to each VM to be executed. The experimental results prove that the proposed architecture keeps the load variation in control and provides better performance of user workload. In experiment a set of 1000, 5000 and 10000 requests are submitted at a time and time taken for identifying the category is negligible. Average execution time for each request is randomly assigned. The average execution time of submitted requests for both SLA and non-SLA members using Strict Differentiated model (SDM) and DLPS is listed in Table 1.

\begin{tabular}{|c|c|c|c|c|}
\hline \multicolumn{3}{|c|}{ Number of Isers } & SD.II & DLPS \\
\hline \multirow[t]{2}{*}{1000} & SLA Member & 400 & $120 \mathrm{~ms}-200 \mathrm{~ms}$ & $100 \mathrm{~ms}-180 \mathrm{~ms}$ \\
\hline & SLA Non-member & 600 & $300 \mathrm{~ms}-600 \mathrm{~ms}$ & $260 \mathrm{~ms}-560 \mathrm{~ms}$ \\
\hline \multirow[t]{2}{*}{5000} & SLAM & 3000 & $900 \mathrm{~ms}-1$ & \\
\hline & SLA Non-member & 2000 & $600 \mathrm{~ms}-900 \mathrm{~ms}$ & $500 \mathrm{~ms}-800 \mathrm{~ms}$ \\
\hline \multirow[t]{2}{*}{10000} & SLA Member & 6000 & $1800 \mathrm{~ms}-2600 \mathrm{~ms}$ & $1600 \mathrm{~ms}-2400 \mathrm{~ms}$ \\
\hline & SLA Non-member & 4000 & $1200 \mathrm{~ms}-1800 \mathrm{~ms}$ & $1100 \mathrm{~ms}-1600 \mathrm{~ms}$ \\
\hline
\end{tabular}

Table 1. Average execution time taken using diffe rential treatment

\section{CONCLUSION}

Nowadays, Cloud Computing has becoming an emerging and fascinating model for outsourcing various IT needs of the organization. At present, there are several cloud providers offering different services with different Quality of Service and SLAs. In addition to that, service providers also increasing wide range the in the world, this leads ambiguity and distrust among the users to select the suitable service provider which can satisfy their QoS requirements in terms of Service Measurement Index suggested by Cloud Service Measurement Index Consortium (CSMIC). The aim of this consortium is to define each of QoS attributes given in the framework, provide a methodology for computing the index attributes for Cloud services and rank them. Therefore many authors proposed different ranking frame work to select best service providers based on the user, application and service requirements. In this chapter, two different ranking mechanisms are proposed to sort the providers and select the optimal provider auto matically. Grade distribution ranking model is proposed by assigning the grade for the providers based on the values of SMI attributes, based on the total grade value, providers falls on either Gold, Silver or Bronze. Each category applies the quick sort to sort the providers and find the provider at the top is optimal. If there is more than one provider at the top, apply priority feedback based decision tree and find the optimal provider for the request. In the second ranking mechanis $\mathrm{m}$, joint probability distribution mechanis $\mathrm{m}$ is used to rank the providers, two providers having same score, apply priority feedback based decision tree and find the optimal provider for the request. Simulation results shows that the proposed ranking mechanism provides better performance compared to the existing mechanis ms such as fuzzy logic set, regression tree and point care method. 


\section{REFERENCES}

[1]. S.K.Garg, S. Versteeg, andR. Buyya, "A framework for ranking of cloud computing services," Future Generation Computer Systems, vol. 29, no. 4, pp. 1012-1023, 2013.

[2]. C.s. rajarajeswari, $2 \mathrm{~m}$. Aramudhan "ranking of cloud service providers in cloud" journal of theoretical and applied information technology, august 2015. Vol.78. No.2.

[3]. P., Metri, G., Sarote (2011).Privacy Issues and Challenges in Cloud Computing, International Journal of Advanced Engineering Sciences and Technologies (IJAEST), vol. 5, pp.1-6.

[4]. IBM (2010), Cloud Deployment and Delivery Models, Available from https://www.ib m.co m/developerworks/my developerworks/c2028fdc [Accessed13July -2011].

[5]. Chenguang he, Xiaomao fan, Ye li (Jan 2013), Toward Ubiquitous Healthcare Services With a Novel Efficient Cloud Platform, Biomedical Engineering, IEEE Transactions on, Vol. 60, no., pp. $230-$ 234.

[6]. Ahn, Y.W.; Cheng, A.M.K.; Baek, J.; Jo, M.; Chen, H.-H (2013). An auto-scaling mechanis $\mathrm{m}$ for $\mathrm{v}$ irtual resources to support mobile, pervasive, Realtime healthcare applications in cloud, Network, IEEE, Volu me: 27, pp.: $62-6$

[7]. Al Iqbal, R (2012). Hybrid clinical decision support system: An automated diagnostic system for rural Bangladesh. Informatics, Electronics \& Vision (ICIEV), 2012 International Conference on, Page(s): $76-81$

[8]. Ilayaraja, M. ; Meyyappan, T. (2013).Mining medical data to identify frequent diseases using Apriori algorithm, Pattern Recognition, Informatics and Mobile Engineering (PRIME), 2013 International Conference on, Page(s): 194 $-199$.

[9]. Chauhan, R.; Kumar, A. (2013), Cloud computing for improved healthcare: Techniques, potential and challenges, EHealth and Bioengineering Conference (EHB), 2013, Page(s): $1-4$

[10]. Huang, Feixiang ; Wang,Shengyong ; Chan,Chien- Chung(2012), Predicting disease by using data mining based on healthcare information system, Granular Computing (GrC), 2012 IEEE International Conference on, Page(s): 191 $-19$
[11]. Saurabh kumar Garg, Steve Versteeg and Rajkumar Buyya, " SMICloud: A framework for comparing and Ranking cloud services" Fourth IEEE International Conference on Utility and Cloud computing, 2011 pp 210-219.

[12]. Princy Bathla, Sahil Vashist, "SLA Aware Cost based Service Ranking in Cloud Computing", International Journal of Application on Innovation in Engineering and Management, Vol.2 Issue 7 Ju ly 2014

[13]. Buyya, R., Ranjan, R., \& Calheiros, R. N. (2009). Modeling and Simulation of Scalable Cloud Computing Environments and the CloudSim Toolkit: Challenges and Opportunities. Proceedings of the 7th High Performance Computing and Simulation Conference, Germany. Leipzig, Germany (pp.1-11). doi:10.1109/HPCSIM.2009.5192685

[14]. Buyya, R., Ranjan, R., \& Calheiros, R. N. (2010). InterCloud: Utility-oriented federation of Cloud computing environments for scaling of application services.10th International Conference on Algorithms and Architectures for Parallel Processing, Busan,

[15]. K. Mohan and M. Aramudhan, aDepartment of Computer Science and Engineering, Sathyabama University, Chennai, India; bPKIET , Karaikal, India, Broker based trust architecture for federated healthcare cloud system, Intelligent Automation \& Soft Computing, 2016 TASJ 1220118, 